\title{
PHOTOMETRIC AND POLARIMETRIC OBSERVATIONS OF \\ THE RV TAURI STAR AR PUPPIS
}

\section{A.V.Raveendran and N.Kameswara Rao Indian Institute of Astrophysics Bangalore 560 034, India}

RV Tauri stars are variable yellow supergiants and they display anomalous excesses in infrared radiation indicating the presence of $\mathrm{coOl}$ and extended dust thermospheres, presumably formed from the matter ejected by them (Gehrz 1972). AR Pup, recognised as a member of RV Tauri stars, shows strong $\mathrm{CH}$ and $\mathrm{CN}$ bands in the optical spectrum and is considered to be carbon-rich (Lloyd Evans 1974). During a programme of broad band polarimetric observations of carbon variables, AR Pup was found to exhibit a large and highly time-dependent linear polarisation (Raveendran \& Kameswara Rao 1987).

During the period from 1987 January 20 to April 6, AR Pup was observed on 33 nights through standard $B$ and $V$ filters with the $34-\mathrm{cm}$ reflector of the Vainu Bappu Observatory, Kavalur. We also obtained UBVRI polarimetry of AR Pup with the PRL - Polarimeter (Deshpande et al. 1985) attached to the $102-\mathrm{cm}$ reflector on six nights during the same period. Present observations clearly indicate that the large time dependent variations in polarisation, both in the amount and wavelength dependence, observed at earlier epochs by us were not isolated events but were segments of a continuous variation which is cyclic and related to the light curve.

In Fig.1, we have plotted the results of BV photometry and the linear polarisation in $V$ band against the corresponding Julian days of observation. It is evident from Fig.1 that polarisation increased during the ascending branch of the light curve, a case qualitatively very similar to what is observed in the long period variable o cet (cf. Shawl 1975). The rapid increase in polarisation is probably related to the outward passage of an atmospheric shock. We find that larger changes in polarisation and position angle occur in ultraviolet than in red. The nature of wavelength dependence of polarisation and rotation of position angle with wavelength seen in AR Pup indicate that the main mechanism which produce the observed polarisation is grain scattering. Since the position angle of polarisation is not constant in time, there is no fixed axis of symmetry for the scattering material. This would suggest that a major portion of polarisation in AR Pup arises from more localised transient regions and not from the extended infrared emitting circumstellar envelope.

The photometric data indicates a $75 \pm 1$ day period for the light variation. 


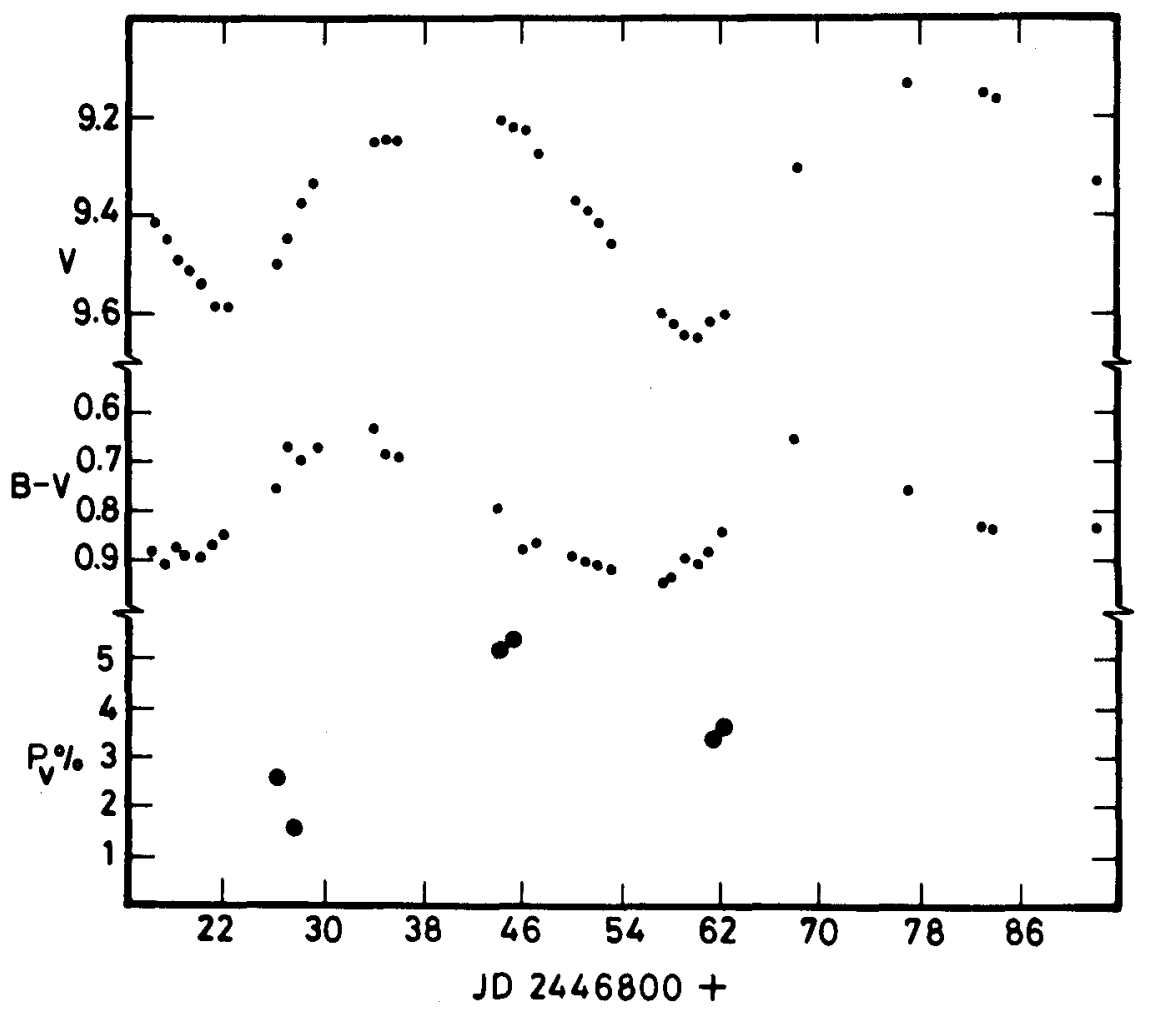
Fig. 1. Plots of $V,(B-V)$ and linear polarisation in $V(P, Y)$
against the Julian days of observation.

A detailed version of the paper will be published elsewhere.

\section{References}

Deshpande, M.R., Joshi, U.C., Kulshrestha, A.K., Banshidhar, Vadher, N.M., Mazumdar, H.S., Pradhan, S.N. \& Shah, C.R., 1985. Bull. Astr. Soc. India, 13,157 .

Gehrz, R.D., 1972. Astrophys. J., 178, 715.

Lloyd Evans, T., 1974. Mon. Not. R. Astr. Soc. 167, 17p.

Raveendran, A.V. \& Kameswara Rao, N., 1987. Astron. Astrophys. (accepted).

Shawl, S.J., 1975. Astron. J. $\underline{80}, 602$. 